ounces of milk, two ounces of beef-tea, and half an ounce of whisky) are administered during this time. On the third day a movement of the bowels is secured by the administration of an enema (one pint of salt water). On the fourth day the gauze drain is removed and care must be taken in doing this not to pall out or disturb the tube, through which a long strong probe may be inserted to prevent kinking whilst the outer end of it is steadied and held in place. On the sixth day, if the discharge is quite small in quantity, the tabe can be withdrawn for half its length and cut off. In another few days, depending upon the amount of discharge, it may be altogether dispensed with.

A fæcal discharge from the wound is usually preceded by a rise in temperature. Neither require any special treatment unless in exceptional instances (Case 3), and the wound heals steadily if kept clean by frequently changed dressings. If the appendicular abscess has occupied a position in the abdomen internal to the cæcum drainage should be aided by tilting the patient over towards the right side. This is readily and comfortably done by placing a pillow lengthwise under the left side of the back when the patient is moved off the operating table into bed. No abdominal belt is needed after the wound has healed.

The mortality of this operation is small, and I cannot recall a single instance in which death could be attributed to it. At the present time and for this part of the country I would give the mortality of appendicular abscess at 8 per cent. of operation cases. This will diminish in the same remarkable way as that for strangulated hernia has done so soon as practitioners become more skilled at early diagnosis and realise more fully the need for operative treatment.

List of Cases of Abscess in Connexion with the Appendix Vermiformis.

\begin{tabular}{|c|c|c|c|c|}
\hline Sex. & Date. & Condition. & Appendix. & Result. \\
\hline & 1899. & & & \\
\hline F. & July 18th. & Abscess. & Removed. & Recovered. \\
\hline M. & August 6th. & $"$ & , & , \\
\hline F. & $" 14$ th. & Pelvic abscess. & $\begin{array}{l}\text { Not } \\
\text { removed. }\end{array}$ & ", \\
\hline M. & Oot. 5th. & Abscess. & Removed. & " \\
\hline F. & $" 10 \mathrm{th}$ & Pelvic abscess. & $"$ & $"$ \\
\hline M. & "25th. & $\begin{array}{c}\text { Abscess with pelvic } \\
\text { peritonitis. }\end{array}$ & $"$ & ", \\
\hline M. & Nov. 7th. & Abseess. & " & " \\
\hline M. & "7th. & 3 & , & , \\
\hline M. & "16th. & ", & " & $"$ \\
\hline M. & Dec. 13th. & ", & $"$ & ", \\
\hline M. & $\begin{array}{l}\text { " 23rd. } \\
1900 .\end{array}$ & ," & , & " \\
\hline F. & Jan. 18th. & "' & , & $"$ \\
\hline M. & " 29th. & $\begin{array}{c}\text { Abscess and pelvic peri- } \\
\text { tonitis. }\end{array}$ & $"$ & " \\
\hline F. & Feb. 14th. & Abscess. & $"$ & 3 \\
\hline M. & , 21st. & $"$ & $"$ & ", \\
\hline F. & , 24th. & $"$ & " & $"$ \\
\hline F. & May 7th. & ", & ", & $"$ \\
\hline F. & "9th. & $\begin{array}{l}\text { Abscess and suppura- } \\
\text { ting ovarian cyst. }\end{array}$ & $"$ & $"$ \\
\hline M. & $" 10$ th. & Pelvic abscess. & $\begin{array}{l}\text { Not } \\
\text { removed. }\end{array}$ & $\begin{array}{l}\text { With a } \\
\text { sinus. }\end{array}$ \\
\hline M. & "14th. & Abscess. & Removed. & Recovered. \\
\hline F. & " 26th. & $"$ & $"$ & $"$ \\
\hline M. & June 21st. & Abscess $\begin{array}{c}\text { with perito- } \\
\text { nitis. }\end{array}$ & " & , \\
\hline M. & , 29th. & Abscess. & " & $"$ \\
\hline F. & July 27th. & " & $"$ & $"$ \\
\hline M. & ," 29th. & " & $"$ & $"$ \\
\hline F. & August 7th. & $"$ & $"$ & $"$ \\
\hline
\end{tabular}

Conclusions.

The early diagnosis of abscess in connexion with the vermiform appendix is of great importance.

A dangerous abscess may be present without any of the ordinary symptoms or signs of pus formation.

The diagnosis is based upon the history of an acute attack of appendicitis and the presence of a definite tender lump.
The position of the appendix and the relations of the abscess may be foretold by a careful study of the tumour.

The diagnosis of pelvic cases in women is attended by special difficulties. In men and women bimanual examination may clear up an otherwise doubtful case.

Spontaneous relief and possible cure may follow the discharge of pus into adjacent bowel.

An abscess, though of appendicular origin, may be remote from the appendix and may be residual. Many of such pus collections are due to the localisation of a general peritoneal infection which bas been recovered from.

Larly operation is the proper treatment and with few exceptions the vermiform appendix should be removed at the same time as the abscess is drained (pelvic cases form the chief exception to this rule).

The abdominal opening should be large enough to permit of perfect inspection of all manipulation and the abscess should be drained from behind.

To Mr. G. G. Turner, surgical registrar to the Royal Infirmary, Newcastle, I am indebted for an appended list of all cases operated upon by me at the infirmary and private hospital in which pus was found in concexion with the vermiform appendix during the past consecutive working 12 months. I am also indebted to him for the notes of the cases related. Mr. W. G. Richardson, assistant surgeon to the Royal Infirmary, Newcastle, has kindly supplied me with drawings made by bimself to illustrate the positions of the varieties of appendicular abscess and the different steps of the operation described.

Newcastle-on-Tyne.

\section{POINTS IN THE CLASSIFICATION AND DIAGNOSIS OF SOME JOINT AFFECTIONS.}

\section{BY GILBERT A. BANNATYNE, M D. GLASG.,} M.R.C.P. EDIN.

HONORARY PHYSICIAN TO THE ROYAL MINERAL WATER AND ROYAL UNITED HOSPITALS, BATH.

DURING the course of a year in Bath, as in most other balneological resorts, one is brought face to face with many difficult problems in respect to the classification and diagnosis of joint affections. And this is comprehensible when one considers how little is really known as to the etiology and pathology of most of them. I am writing this paper with the idea of drawing attention to certain points which have struck me as of importance and value, and as being of interest to others working in the same branch of medicine and also with the hope that by the exchange of views we may be enabled to come to some common understanding on various vexed points. For the present no two men seem to agree as to the diagnosis in certain classes of disease; for example, and to show how chaotic our ideas are, take that most common generic name "rheumatic gout." To judge from the number of patients labeled as suffering from that disease one would think it was the most common of all joint affections; yet what is the nature of "rheumatic gout"? Is it gout or rbeumatism, rheumatism in a gouly subject, gont in a rheumatic, a disease $p e r$ se, or rhenmatoid arthritis? Such are a few of the questions which arise on seeing a case so labeled. On investigation such cases almost invariably can be placed in some more definite and scientific category than that of "rhenmatic gout," whose one virtue seems to be that it covers a multitude of sins and errors in diagnosis, but whose entity as a separate disease is doubtful. This looseness in diagnosis in all joint affections arises not so much from want of knowledge or care on the part of those making the diagnosis as from the confusion of ideas which exists, more or less, in all our minds as to certain forms of disease. On reference to most textbooks one is struck by the unanimity with which joint diseases are described as they have been described for years, irrespectively of the advance of science, and the absence of method in classing them according to their mode of origin. Now it seems to me that most arthropathies can be referred to several great classes : (1) the bacterial or toxic arthropathies; (2) the nerve arthropathies; and (3) the senile degenerative arthropatbies. But besides placing all arthritic cases in these classes it is also possible further to 
differentiate between the essential arthropathies-i.e., those in which the joint troubles form the principal symptom of the disease, and the accidental arthropathies -i.e., those which occur with a certain amount of regularity but are not essential symptoms of the disease in which they arise. Thus I should class as essential arthropathies rheumatism, rheumatoid arthritis, gout, senile arthritis, and pulmonary osteo-arthropathy, whereas gonorrhœai, scarlatinal, malarial, and nerve arthropathies are accidental but integral symptoms of some specific condition. I should also explain that by the expression "toxic" I do not wish to confine the meaning of the word to the products of bacterial activity only, but desire to include all products, whether elaborated by specific bacteria or arising from the tissues themselves or by chemical processes. Thus, I am enabled to place gout in a class with other diseases presenting many similar points of interest.

In studying joint diseases one sees how opinion has altered with regard to their origin. A few years ago the nervous origin of most of them was firmly believed in, yet now they can, to a great extent, be marshalled into the bacterial or toxic category. Whilst admitting that pure nerve arthropathies exist, yet from what we know of bacterial activity it certainly seems more reasonable to suppose that they or their products are the cause of most joint changes, rather than that some obscure nerve degeneration or reflex action should be so. As the specific cause in more and more diseases is found to be bacteria or their products, so does it become more likely that such micro-organisms are the cause of nearly all of them.

According to our present knowledge the more common arthritic troubles may be classed as follows:-1. Bacterial or toxic arthropathies: (a) bacterial-rheumatism, rheumatoid arthritis, gonorrhoeal and scarlatinal arthritis (and probably malarial arthritis); and (b) toxic-gont and pulmonary osteoarthropathy. 2. Nerve degenerative arthropathies, such as occur in tabes, ataxic paraplegia, \&c. 3. Senile degenerative arthropathies, such as senile arthritis or malum coxa senilis. Malarial arthritis may be classed provisionally as bacterial, for I take it that the malarial parasite may be regarded in its action as to all intents and purposes a bacterium, although differing considerably in its life-history.

Another point to notice is that the joint changes in any of the above complaints are not complications, but are all true manifestations of their separate disorders. They are not secondary or separate infections or diseases, but some doubt may still exist whether in a disease having a bacterial causation the bacteria themselves or their products are the canse of the symptoms. Some observers hold that it is the products which give rise to them, whilst others maintain that it is the micro-organisms themselves, and with these latter I side myself. What evidence we have is in favour of this view; for have not the micro-organisms of rheumatism, rheumatoid arthritis, and gonorrboea been found in the joint structures and joint fluids? In diseases having constant and well-marked changes it is perhaps only natural to expect that the materies morbi itself should be found at the seat of greatest mischief. And if the changes were only caused by products elaborated elsewhere surely they would be much more amenable to treatment, more evanescent in their course, and more unstable in their character. And what better nidus could be found for any micro-organic growth than a joint, surrounded by a fluid medium richly-endowed with nutriment suitable to their growth ? No, I cannot but feel that most forms of arthritis, whether the primary and principal manifestation of a disease or secondary and unimportant, must have bacteria as their provocative agent. So far science, in some conditions, has not found the bacterial agent. but all the same the infective character of most of them is manifest, and so, until more perfect and fuller knowledge is ours, one must rest content.

In considering varions points of interest in the various diseases I have attempted to classify, one's attention is first turned to rheumatism. It is a big field and a big question for observation, but is much simplified if we consider all its forms and modifications as only so many different manifestations of one and the same poison. If this is not done, and if we look on them as due to secondary or separate infections $-a$ condition of things of which there is no proof-a very large question for speculation is opened out. So far all positive proof lies on the side of one poison capable of affecting many and various structures, and from this standpoint I wish to study certain points.

The first point $I$ wish to direct attention to is the ${ }_{\mathrm{p} .26}^{2}$ occurrence of swellings or nodules in various stractures-of with considerable frequency. The first of these is seen in muscular rheumatism, a disease which at one time was regarded as possibly a separate disorder, but which now, I think, is generally acknowledged to be only one of the manifestations of the rheumatic poison. In this form of rheumatism, especially in those who have had repeated attacks, one's attention is frequently attracted to masses or indurated patches in certain muscles pointing to an inflammatory infiltration of the muscle substance. These patches in themselves give no pain and the patients themselves are usually unaware of their presence. The masses are the result of an interstitial myositis, probably brought on by toxic action-at least, that is the idea of those who have studied the question (Leube and Adler). ${ }^{1}$ Patients who present these changes not infrequently develop later arthritic changes and endocarditis. These patches are distinct from the subcutaneous nodules so often noted in rheumatism, rheumatoid arthritis, gout, \&c., and which appear to be entirely formed of fibrous structure, thus differing from those already mentioned. These subcutaneous nodules have been looked on as being of important diagnostic value, as it was at one time thought that they only occurred in rheumatism and that therefore all cases in which they were seen were cases of that disease. But they have been observed fairly often in rheumatoid arthritis and they occur less frequently in gout, syphilis, influenza, \&. Dr. Hawthorne ${ }^{2}$ whose able paper on this subject has done much to bring the question prominently before the profession, contends that the mere presence of such tumours is no proof of rheumatism. With this I fully agree, for numerous instances of subcutaneous nodules have come under my observation in gout and rheumatoid arthritis and once in gonorrhœeal arthritis. They also occur in Maltese fever, a disease which often presents arthritic changes. As one would expect from the nature of the disease, those seen in rheumatism are more transient in nature and smaller than those seen in rheumatoid arthritis. In this latter complaint they are most often seen in the chronic and later stages.

While on this subject there are yet two other forms of swellings in connexion with arthritis which well repay study. One of these is due to the swelling of bursæ in connexion with joint affections and is most frequently seen in chronic gout or chronic rheumatoid cases, and occasionally in rheumatism. Many of these swellings are merely enlargements of normal bursæ and having an intimate connexion with a joint, whilst others have apparently no connexion whatever with one and may even be situated at some distance from any joint. Both kinds are liable to chronic inflammatory changes and may even contain foreign bodies of a cartilaginous nature. They seldom suppurate-never in rheumatoid arthritis-and only as the result of secondary pyogenic infection. I remember one marked case occurring in rheumatism, the patient being a woman, and who, subsequently to her second attack of acute rheumatism, developed an enlarged bursa over the left olecranon. The bursa enlarged rapidly, causing considerable pain, but on treatment subsided almost as rapidly. Some months later she had another attack of rheumatism, less acnte than the other, during which she had a similar enlargement on the other side, and which also subsided rapidly. The other and last class of nodular enlargement to which $I$ wish to draw attention is that described by Heberden This form has often been considered to be merely a form of rheumatoid arthritis and to be only seen in that disorder. But, common as it may be in chronic rheumatoid arthritis, I have seen nearly as many in chronic gout. Of this $I$ am certain-these nodes are osseous in character and arise principally from the ends of the phalangeal bones. They are permanent in nature, although sometimes an apparent diminution may be noted under treatment owing to the superimposed tissues being reduced in size.

It is of interest to consider the development and diagnostic value of these nodular enlargements, but with regard to the latter I fear it is small, and this is obvious when we consider that they must all originate through some irritation of the different structures involved. This irritation may be due to micro-organic activity or it may be due to some toxic action, but the result is the same-namely, a chronic interstitial

1 Medical Record, 1900 , lvii., p. 529.

Rheumatism, Rheumatoid Arthritis, and Subcutaneous Nodules, 
change, resulting in one case in bony nodes, in another in bursal enlargements, in another in fibrous nodules, and in yet another in muscular masses. The explanation of these manifestations is therefore simple. Dr. F. J. Poynton and Dr. A. Paine having isolated their diplococcus in rheumatic nodules strengthens my chain of argument.

As aids to diagnosis I should say muscular swellings are most often due to rheumatism, small subcutaneous nodules also to rheumatism, larger ones to rheumatoid arthritis, bursal enlargements to chronic gout, rheumatoid arthritis, or rheumatism, and bony nodes to chronic gont or chronic rheumatoid arthritis.

Rheumatism has long been looked upon as an infective disease, but many and various have been the micro-organisms found. The researches of Dr. Poynton and Dr. Paine ${ }^{3}$ go far to settle the question, but they are not conclusive, for it is too early to say whether the organism isolated by them is the only cause of rheumatism or whether it is only one of several which may give rise to rheumatic symptoms; and while we are still much in the dark as to mixed infections and what influence one micro-organism has upon another in determining its virulence and mode of growth, from the nature of the symptoms seen in rheumatism I feel sure only one organism is the cause. It seems evident that if a certain one is a constant feature in a certain disease or group of symptoms it must have some im. portant bearing in its causation. A fact of importance from my present point of view is that Dr. Poynton and Dr. Paine have been able to demonstrate the presence of their diplococcus in the joint fluids and structures as well as in other sites and by inoculation have been able to reproduce the disease in animals. The inoculations gave rise to arthitis, bursitis, and teno-synovitis, as well as other symptoms, and from each of these inflammations they were able to isolate again the same diplococcus. The chain of evidence seems to me complete, but whether their organism will prove to be the only cause in all cases of pure rheumatism future scientific investigation alone will show. I cannot think that a disease with such well-marked and constant symptoms can be caused by more than one species of bacterium, although this one must have varying degrees of virulence, and its action must be governed by the attacked individual's power of resistancethe different sets of symptoms arising through the fact that the structures affected are influenced by those peculiar forces " natural selection" and "susceptibility of parts." What those forces are is not known, but it is a wellrecognised fact that the various tissues of the body respond in different ways to different causes of disease, and that the same cause will affect different structures at different times under apparently similar circumstances, and even in the same person, in different ways.

On the subject of rheumatoid arthritis I feel strongly. One of the principal points in this disease which strikes an observer is that apparently under this name we have two conditions, one acute and the other chronic. The acute form of the disease is seen principally amongst children and in the comparatively young. It is not a common disease of later middle life or of old age. It is more common in females than in males and is the sequela, as a rule, of some other infective disorder, such as influenza, rbeumatism, or tonsillitis. It is accompanied by a rise in temperature and swelling of the joints, which become tense from synovial distension or else soft and doughy, but with no cartilaginous or bony outgrowth; in fact, the joint presents an almost pathognomonic soft spindleshaped enlargement. The extremities are cold and sweating and present a livid mottling, while numerous small freckles or patches of pigment appear on the skin and there may also be other vaso-motor or trophic changes. There may be great pain or almost none. Mnscular atrophy is always present and shows marked selection in the muscles or group of muscles affected. Glands proximal to the affected joints are of ten enlarged, whilst anæmia may be a marked feature. Patients show a loss of fat and vital energy and usually present a peculiar facies which once seen is seldom forgotten. On the other hand, in the chronic cases, which may be chronic from the first or else which may be the later stages of an acute attack, the joints show a progressive crippling which becomes more and more marked as the disease advances. It is most frequent in middle life and in females, is ascompanied by no fever, and the general

8 The Larcer, Sept. 22nd (p. 861) and 29th (p. 932), 1900. health suffers little; it may affect only a few joints but more often many; it is very chronic in its course but is progressive with exacerbations. If chronic from the outset it usually begins in one small joint as a slight enlargement, which in time increases in size, and the disease then spreads to other joints until it may become difficult to move any joint, from stiffness, pain, and deformity. It is accompanied by much crepitation, usually of the coarser variety, and cartilaginous and even bony outgrowths appear round the margin of the joints as well as in the tendons, bursæ, \&c. The movements of the limb may be much interfered with by adhesions, bands, and thickenings, whilst, in very advanced cases, even dislocations may occur. As a sequela of an acute attack, as the acute symptoms subside, the joints become more irregular, more crippled, and in fact come to present all the characteristics just described. It is not within the province of this paper to consider the pathological changes in the joints, but I would mention that the main points are, in the acute form, an inflammatory condition marked by nlceration and destruction of the joint tissues with no outgrowth or lippings, whilst the chronic form is characterised by hardening and thickening and with cartilaginous and osteophy tic outgrowth and lippings.

I have so often insisted on the bacterial origin of the disease that I will not do so further here, beyond mentioning that it may be of interesi to some to know that some little time ago Von Dungorn and Schneider ${ }^{4}$ succeeded in isolating a small micro-organism which, on injection, reproduced the disease in animals. Their micro-organism was found in the joints and gall-bladder. I have not had an opportunity of comparing it with the micro-organism found by Dr. A. S. Wohlmann and myself, but it helps to prove that the disease is due to bacterial infection. Further scientific research will show whether it is due, as I believe, to one specific bacterium and that one the one found by Dr. Wohlmann and myself $\overline{ }{ }^{\bar{b}}$ or to otber and mixed infections. As in the case of rheumatism we must wait for further proof.

The other point with regard to rheumatoid arthritis that I wish to touch on is the muscular atrophy so often seen. There are seemingly two varieties, one, exhibiting a selective affinity in its distribution for certain muscles or group of muscles, is seen in the acnte forms; the other, as seen in chronic cases, being apparently merely due to disuse. In the acute form the atrophy is so acute and occurs so soon after the onset of the disease and whilst the joint changes, may be, are extremely slight and is so selective in character (the extensors and interossei being principally, but not exclusively, affected) as to preclude the possibility that it is the result of disuse only. It may affect one muscle only or a whole set, but it affects the whole muscle and not a part of it only. It of ten affects muscles proximal to the joint disease and the tendon reflexes are in most cases exaggerated. In the chronic cases the atrophy affects only those muscles not employed and in direct ratio to the amount of disuse and there is no increase in the tendon reflexes. It has been argued that the form seen in the acute cases, especially by reason of the exaggerated reflexes, lends conclusive support to the dystrophic theory of the origin of rheumatoid disease. But this does not follow, for we know other arthritic lesions may produce muscular wasting having similar characters. All it proves is that this particular form has all the characters of an atrophy due to nerve (probably spinal) irritation. Some hold that this irritation is set up by reflex action, but to my mind it is more probable that it is the result of toxic poisoning. We have no proof that bacteria themselves act on the central nervous system, but the whole nervous system seems to be peculiarly liable to the action of their poisons. For example, take the case of diphtheria or tetanus; or, again, certain toxins are known to act on the vaso-motor system and also on the trophic nerve condition of various structures. Thus tachycardia has been caused, according to Bezançon, by toxins. Bouchard obtained from ruberculin a substance causing dilatation of the blood-vessels, whilst Charrin and Gley obtained toxins with similar properties from the bacillus pyocyaneus, as also did Arloing from the staphylococcus aureus. Vier $r$ rt has reported a case of phthisis with neuritis of the vagus, and Semmola mentions the action of the influenza toxins on the vasomotor centre. In no case of rheumatoid joint disease do the nerve troubles precede the joint manifestations; therefore they must in all probability arise secondarily 5 THE LANCET, April 25th, 1896, p. 1120. 
to them. But it has been argued that if muscular atrophy can, or does, arise through reflex impulses, so also may some peripheral irritation, say, from such an organ as the uterus, cause impulses capable of originating not only the muscular atrophy but also the joint lesions. This I cannot agree with. I do not deny that it is possible for reflex impulses to alter the nutrition of certain muscles and thus atrophy, but that they can give rise to grave organic changes of an acrite and severe character seems to me impossible without at the same time producing some alterations in the nervous centres or in the nerves themselves easily recognisable on microscopic examination. Such changes have not been found, and until they are I cannot believe that reflex irritation alone can cause joint changes of the character seen in rheumatoid disease. In this disease, unless distinct nerve le:ions are proved to exist, the theory of infection seems the more probable, the more easily understood, and the more true to clinical experience.

Gonorrhce 1 arthritis presents several points of interest fron a diagnostic standpoint. The arthritis is always secondary to a gonorrhœal attack elsewhere, but it does not always imply that the primary attack is in the urethra. A considerable number of cases have followed infection of the conjunctiva, and this is peculiarly noticeable in children. The joint troubles are caused by the presence of the gonococcus in the joints, and it can be isolated and cultivated from the joint fluids. It is only found, however, with certainty in the early stages of the arthritis. The time of onset of the joint changes may vary: sometimes they occur within a week of the infection, but more often not until after three or four weeks. Cases have even been known to occur many months after the primary infection and when only a slight gleet has existed at the original seat of inoculation. The severity of the joint troubles, therefore, has no relationship to the severity of the primary trouble. It is most liable to affect the larger joints, especially the knees and the joints of the lower limbs rather than those of the upper. For the severity of the symptoms there is an indication in the great effusion of fluid, but the skin is seldom reddened. The pain experienced is not so severe as in a similar condition due to rheumatism. Cardiac disease is rare but not unknown. I remember well a case which in many respects resembled one of ulcerative endocarditis, and the nature of the disease was not cleared up until the occurrence of arthritis in both knee-joints many weeks after the patient was brought under observation. This case was also rather peculiar in the fact that the gleet was of the very slightest and that the joint troubles only came on as the temperature was falling and as the cardiac and general symptoms were improving. As a rule a gonorrhoal discharge renders the diagnosis easy, but in women this may not be so, as the import of a vaginal discharge may readily be overlooked or misinterpreted. It is most often mistaken for true rheumatism but the comparative absence of fever and its refusal to yield to the salicylates usually point out its true nature. Sometimes it may be confused with rheumatoid disease, more especially should it affect, as it may rarely do, the ternporo-maxillary joints. It must not be forgotten also that in gout there may be a urethral discharge of a nonspecific nature. However, as a rule, the joint trouble is so distinctive as to lead to little or no confusion.

Scarlatinal arthritis is a type of arthritis seen in several other infectious diseases. It is usually seen in severe cases and in adults and appears most often just as the rash is disappearing. It may affect several joints and may be so severe as to suppurate, but this is not common. The elbows and knees are the joints most frequently affected, and it has a greater tendency to affect the smaller rather than the larger joints and those of the upper rather than those of the lower extremities. As a rule the disease yields rapidly to the salicylates. This infectious form of disease is not one of ten seen at a balneological resort, but now and then we come across cases which have their origin in the poisons of infectious fevers and which have persisted for long periods. It is also of interest on account of the frequency with which scarlatinal and rheumatic patients develop chorea, possibly pointing to an intimate relationship between the two diseases. If we accept the discovery of a diplococcus (by Dr. W. J. Class ${ }^{6}$ ) as the specific cause of scarlet fever one is tempted to ask what is the relationship of this diplococcus to that of rheumatism as described by Dr. Poynton and Dr. Paine.
It would be of great interest to know what their reaction is one upon another and what their influence was on each other's growth and virulence, and what influence they had on the production of chorea. Do their toxins when inoculated into the central nervous system produce choreic movements ? Given a micro-organism as the cause of scarlet fever it is probable that the joint troubles are originated by the organism itself. Where suppuration occurs we know that pyogenic organisms must have reached the joints. If they, why not the others? If suppuration occurs it is almost certainly due to secondary infection and it is in such cases that the streptococcus pyogenes has been found. Class's micro-organism does noú cause suppuration and it seems almost certain that the streptococcus is only an accidental secondary infection.

- Malaria, to turn to a subject which is exciting at present a good deal of interest, is frequently associated with arthritic changes. In malarial cases I have seen joint troubles in two forms - one obviously due to true rheumatism and contracted probably at the same time as the malarial poison; the other form is rarer and occurs apparently as an integral part of a malarial attack. Two cases of the latter variety some time ago presented themselves to me. Both patients were in the army and their malaria had been contracted in Burma. They had never served in the Mediterranean. When first seen there were no arthritic symptoms, but they were both subject to malarial attacks at more or less irregular intervals. They had for some time past been subject to joint pains during these attacks and had therefore come to Bath to see what we could do for them. Soon after coming under observation they had attacks of ague, going through the cold, hot, and sweating stages, but as the temperature fell the patients complained of pains in the limbs and joints, the joints most affected being the knees, elbows, wrists, and ankles. The joints became somewhat swollen and painful and remained so for possibly two or three days. Muscular pains accompanied the joint ones but were not so severe or so marked. There were no cardiac troubles but there was anæmia and splenic enlargement. During the time they were under observation each patient had from three to four such attacks and on each occasion presented somewhat similar symptoms, but towards the end of their term here the arthritic symptoms did not seem quite so marked or so severe. However, they were by no means cured and I found treatment unsatis factory. As the occurrence of joint troubles in malaria is, I believe, somewhat rare, the question arose as to the exact nature and cause of the attacks. In neither case was there any suspicion of Maltese fever and the history of the onset of the disease in Burma was quite distinct. As we know that in Maltese fever joint troubles occur due to the micrococcus melitensis it occurred to me that possibly a somewhat similar condition might exist in Barma, but $I$ can find no history of any such disease. The attacks in my patients differed to what occurs in Maltese fever, where the joints are only affected some time after the onset of the fever and where they are also associated with a good deal of muscular pain and neuralgia.

Another point of interest is that the malarial poison seems to affect adversely the curability of both gout and rhenmatism. These two diseases, when seen in malarial subjects, usually present themselves in most intractable forms. Of ten a gouty attack will determine one of ague and vice versâ. Another thing I have noticed is that in some cases, if quinine be freely administered for the ague, it seems to bring on an acute attack of gout. So much so is this that many patients tell me they can never take quinine without bring ing on gouty symptoms. This is especially marked with the sulphate and hydrochlorate of quinine, much less so with the salicylate. The reason for all this seems quite unknown and I merely mention my own observations in the hope of drawing attention to certain points worthy of further investigation.

A disease, pulmonary osteo-arthropathy, may give rise to some little difficulty in diagnosis, principally owing to its rarity. It is most likely to be confounded with acromegaly, but in the latter disease there is no alteration of the nails nor are the finger-ends clubbed nor the carpus and metacarpus much thickened. The chief characteristics of the disease are great enlargement of the hands, wrists, feet, and ankles, associated with, and secondary to, some chronic pulmonary affection, such as phthisis, chronic bronchitis, and empyema. In the joints the changes are effusion with enlargements and ulceration of the cartilages and articular ends of the bones. Marie is of opinion that these changes 
are due to toxic poisoning, but Thorburn looks on them as tuberculous. The evidence either way is slight and indefinite.

Recent research in gout seems to have done little but upset all our former ideas as to its pathology and origin. Not so long ago it seemed as if we had arrived at some common agreement on the subject, but every division of it has recently been re-investigated, with the result that all our old theories have been found wanting, and the new ones brought forward are so diverse and uncertain that it is apparently impossible to formulate any satisfactory theory. After perusing much literature on the subject $I$ find that about the only fact which seems tolerably certain is that uric acid, or its derivatives, is the immediate cause of the joint troubles. But why uric acid should be deposited in these positions; why there should be an excess of uric acid in the blood, it such excess exists, and why it should not be excreted as is done in other conditions, seem to be still sub judice. It appears to me that something more than excess of uric acid is necessary, and the theory of a toxic condition of the blood or tissues being the determining agent, seems from one's experience to be the most likely solution of the difficulty. All experiments on the healthy subject have failed to bring about the gouty state, and therefore it seems as if in gout the blood or tissues acquired some peculiar characteristic, with the result that some ingredient of the blood which in the natural state would be excreted without any symptoms becomes a poison capable of causing acute joint troubles. So much, however, is pure speculation that I do not care to enter further into the subject bere.

Questions of diagnosis between certain irregular and chronic forms of gout and some forms of rheumatoid arthritis and subacute and chronic rheumatism offer the most common difficulties we who practise at bathing resorts have to consider. The acute forms of the various diseases are so characteristic as to give rise to no trouble. It is as gout passes from the acute into the chronic and more irregular forms, or where it has never been acute, that diagnostic difficulties arise. Especially is this so in middle-aged or elderly women, and it is often only after prolonged and patient observation that a definite diagnosis is possible. Yet from the point of view of treatment this is all important. Gout is more common in men, but a certain number of cases occur in women, usually of a chronic irregular form. It mostly occurs in the well-to-do and well-nourished. In irregular cases it does not occur suddenly, nor is the onset acute, but possibly there is the previous history of such an acute attack. It is seldom symmetrical, almost never affects the temporo-maxillary joints or back of the neck, and is much more liable to affect the joints of the lower limbs than those of the upper. Owing to the deposits of the biurate in the joint structures the latter are often irregular in size and shape, sometimes being nodular, but they may present almost any appearance. There is no tendency to destruction of the tissues. It is often associated with digestive troubles and irritability of temper, as well as marked depression of spirits. The teeth are usually ground down, whilst the nails may be cracked and marked longitudinally.

Chronic rheumatoid arthritis is more common in women than in men, and amongst the poor and badly-nourished. It usually begins insidiously in one of the metacarpo-phalangeal joints and is not associated with redness or great tenderness. It rapidly spreads to many other joints, giving rise to much crippling and deformity. It is specially liable to affect the temporo-maxillary joint and the back of the neck. There are marked muscular atrophy and of ten pigmentary and vasomotor changes. Once a joint is affected there is little tendency to improvement. There is marked symmetry in the joints attacked, but the first symptom can usually be traced to one side. There are no biurate deposits, although lipping and outgrowths may be mistaken for them. The cartilages are ulcerated and there may be much coarse grating. It is not readily affected by the salicylates. Chronic rheumatism never affects the temporo-maxillary joints and is not associated with the formation of bony or cartilaginous deposits in the joints. It exhibits little $\mathbf{8 y m}$. metry and is not progressive. It is usually associated with rheumatic pains and yields to the salicylates in a marked degree.

In certain nerve diseases arthritic changes are noticed and of these tabes may be taken as a type. These changes do not give rise to much trouble in diagnosis, and it is more from the point of view of origin that they are of interest. In them the joint troubles are the result of trophic influences, which alter the joint's nutrition to such an extent as to give rise in some cases tven to acute inflammation, but more frequently only to a chronic condition characterised sometimes by much tenderness, at others by none, by nlceration of the cartilages, by fibrous adhesions, and by alterations in the articular ends of the bones. In all such cases a condition of nerve degeneration exists, the result of some serious organic lesion of the nervous system. This degeneration has been found in every case in which it bas been looked for, and it has even been found in the nerves of the joints themselves. It was the absence of these changes which first threw doubt on the origin of rheumatoid arthritis, and it appears to me that this in itself is so strong an argument that one can positively say that rhenmatoid disease, in the absence of nerve lesions, must originate in some other way.

Senile degenerative arthritis is essentially a monarticular disease, although in some few cases we see two or more joints affected. It is seen in elderly people, especially men, and in most cases as the consequence of some injury more or less trivial. The joint changes are unaccompanied by any vaso-motor or trophic changes, except such as are the result of general malnutrition or disease. Atrophy of the muscles of a limb may of ten be marked, but we rever see any reaction of degeneration, and the muscles usually respond quickly to treatment. This, however, I am sorry to say, is not the case with the artbritis, which is persistent and, if left alone, progressive, ending in the loss of the use of the affected limb. The joints most frecuently affected are the larger ones, especially the hip and the shoulder. The movement of the affected joint being limited and painful, the patient is inclined to save it as much as. possible. There is grating almost invariably and often shortening. There is little or no swelling visible and no redness. Deep pressure always elicits pain, in the case of the hip most pain being felt in the groin and in the shoulder either in front or "on tbe outer side of the joint. The joints show thickening and chronic inflammatory changes of the synovial membrane, whilst the articulating surfaces are worn down, the cartilages being destroyed in patches and the bone being soft and yellowish from fatty degeneration and absorption of its structure. It presents all the characters of a local progressive degeneration.

It will thus be seen that as our knowledge at present stands arthritic troubles arise in a great variety of ways. There are those due to (1) bacteria themselves acting on the joint structures; (2) bacterial poisons (pulmonary osteoarthropathy); (3) toxic poisons of as yet an undetermined character (gout); (4) nerve degenerations; and (5) senile degenerations. Of these Nos. 2 and 3 will probably be referred to some other category as further scientific data become available.

Bath.

\section{ON PROTECTIVE INOCULATION AND SERUM-THERAPY. ${ }^{1}$}

By J. L. BUNCH, M.D., D.Sc., M.R.C.P. LOND.

IN speaking to-night of the subject of serum-therapeutics I am speaking of a subject which is now essentially practical -a subject which is no longer of exclusive interest to the bacteriologist and to the purely scientific worker, but one which is of far-reaching importance both to the medical practitioner and to the patient. Within the last few years the treatment of some of the acute infectious diseases has undergone considerable modification and angmentation, with the result that the prognosis has been greatly improved and the mortality, more especially in diphtheria, to a marked degree diminished.

By the injection of certain antitoxic sera it has been found possible to render less fatal and to cut short the attacks of some of the most dreaded diseases, and even, by the methods of protective inoculation, to render the individual insusceptible to certain öiseases and artificially to confer on him a more or less permanent immunity. Such immunity or power to withstand the attacks of certain infectious diseases which to others are destructive belongs

1901
A paper read before the North London Medical Soeiety on Feb. 14th, 